\title{
BMJ Open Impact of COVID-19 on female fertility: a systematic review and meta-analysis protocol
}

\author{
Fangyuan Li, ${ }^{1,2}$ Hua Lu (D) , ${ }^{1,2}$ Qi Zhang, ${ }^{1,2}$ Xinyun Li, ${ }^{1,2}$ Tong wang, ${ }^{1,2}$ \\ Qianchen Liu, ${ }^{1}$ Qian Yang, ${ }^{1,2}$ Lingxia Qiang ${ }^{1,2}$
}

To cite: Li F, Lu H, Zhang Q, et al. Impact of COVID-19 on female fertility: a systematic review and meta-analysis protocol. BMJ Open 2021;11:e045524. doi:10.1136/ bmjopen-2020-045524

- Prepublication history and additional material for this paper are available online. To view these files, please visit the journal online (http://dx.doi. org/10.1136/bmjopen-2020045524).

Received 04 0ctober 2020 Revised 08 January 2021 Accepted 10 February 2021

Check for updates

(c) Author(s) (or their employer(s)) 2021. Re-use permitted under CC BY-NC. No commercial re-use. See rights and permissions. Published by BMJ.

${ }^{1}$ Hospital of Chengdu University of Traditional Chinese Medicine, Chengdu, China

${ }^{2}$ Chengdu University of Traditional Chinese Medicine, Chengdu, China

Correspondence to

Dr Hua Lu;

Ih18980880525@126.com

\section{ABSTRACT}

Introduction The increased social and economic burden caused by the novel COVID-19 outbreak is gradually becoming a worrisome issue for the health sector. The novel coronavirus invades the target cell by binding to ACE2, which is widely expressed in the ovaries, uterus, vagina and placenta. Significantly, the SARS-CoV-2 is said to interrupt female fertility through regulating ACE2. Thus, it is essential to investigate if the novel COVID-19 hampers female fertility, given that there is no systematic and comprehensive evidence on the association of COVID-19 with female fertility.

Methods and analysis We will systematically search cohort studies, cross-sectional studies, case-control studies and self-controlled case series designs in the following databases: Web of Science, PubMed, EMBASE, Cochrane Library, Ovid, EBSCO, WHO COVID-19 Database, Chinese Biomedical Databases, China National Knowledge Internet, VIP and WanFang Database. Medical Subject Headings and free-text terms for "COVID-19" AND "female" AND "fertility" will be performed. Eligibility criteria are as follows: population (female patients aged 13-49 years); exposure (infection with SARS-CoV-2); comparison (population without SARS-CoV-2 infections or latent SARS-CoV-2 infections); and outcome (female fertility, such as ovarian reserve function, uterine receptivity, oviducts status and menstruation status). Article screening and data extraction will be undertaken independently by two reviewers, and discrepancies will be resolved through discussion. We will use the $\mathrm{I}^{2}$ statistics to assess the heterogeneity and perform a meta-analysis when sufficiently homogeneous studies are provided. Otherwise, a narrative synthesis will be performed. We will explore the potential sources of heterogeneity using subgroup analyses and meta-regression.

Ethics and dissemination Formal ethical approval is not required, and findings will be published in a peer-reviewed journal.

PROSPERO registration number CRD42020189856.

\section{INTRODUCTION}

The national and global spread of COVID-19 caused by SARS-CoV-2 has made it one of the most severe public health threats. Globally, as of 2 September 2020, there have been 25602665 confirmed cases of COVID19, including 852758 deaths, reported by

\section{Strengths and limitations of this study}

- The present systematic review will follow the Preferred Reporting Items for Systematic Reviews and Meta-Analyses guidelines.

- The present study has an established aim, stringent inclusion and exclusion criteria, and a precise quality evaluation and quantitative synthesis.

- It will assess the evidence for differential impact of COVID-19 on reduced fertility risk in different population subgroups, for example, by different ages and the severity of disease through subgroup analysis.

- Two reviewers will independently screen for eligibility and data extraction, with a third reviewer mediating when a disagreement arises, thus ensuring that reviewer bias is minimised.

- The lack of uniformity in measures of fertility may yield significant heterogeneity.

the WHO. ${ }^{1}$ SARS-CoV-2 infection disrupts normal immune responses, ${ }^{2}$ leading to the local and systematic damage of tissues. ${ }^{3}$ Local tissue damage primarily affects the lower respiratory tract and presents as pneumonia, including fever, cough, expectoration and haemoptysis. ${ }^{4}$ Extrapulmonary damage of COVID-19 includes acute kidney injury, hepatocellular injury, neurological illnesses, myocardial dysfunction and arrhythmia, and gastrointestinal symptoms. ${ }^{5}$

Besides, sperms are also susceptible to viral attack $^{6}$ and SARS-CoV-2 infection may most likely cause male infertility. ${ }^{7}$ SARS-CoV-2 appears to have shown adverse effects on the reproductive system. Some studies have shown that SARS-CoV-2 might affect female fertility and disturb female reproductive functions. ${ }^{89}$ Given the scale of the COVID-19 pandemic, there appears to be a potential decline in fertility. ${ }^{10}$ A study reported that many young adults have sexual and reproductive health problems due to COVID-19 pandemic and related containment measures. ${ }^{11}$ It has been reported that COVID-19 is usually accompanied by high levels of interleukin (IL)-6, 
IL-8, tumour necrosis factor- $\alpha$ and other cytokines, which trigger a procoagulant state that is unfavourable to the development of blastocyst or fetus in a normal human uterus. ${ }^{12}$ An epidemiological study demonstrated that coronaviruses could have adverse effects on fetuses and infants, including preterm delivery, intrauterine growth restriction, spontaneous abortion and even death. ${ }^{13}$ Moreover, studies have documented the presence of SARS-CoV-2 across the placenta even in pregnant patients with mild COVID-19 disease, potentially leading to fetal growth restriction and other pregnancy complications. ${ }^{14}$ Accumulating evidence now suggests that 2019-nCoV/ ACE2 may interfere with the female reproductive functions, leading to menstrual disorder, infertility and fetal distress. $^{8}$

ACE2 is a receptor for SARS-CoV ${ }^{15}$ SARS-CoV-2, as a subgenus Sarbecovirus of the genus Betacoronavirus, shares a $76 \%$ amino acid sequence homology with SARS-CoV. ${ }^{16}$ The protein expression profile of ACE2 is also considered to be the host receptor of SARS-CoV-2. ${ }^{17}$ Thus, SARS-CoV-2 can invade target host cells by using the ACE2 as the primary receptor binding site ${ }^{18-20}$ and regulate the expression of ACE2 in host cells. ${ }^{8}$ ACE2 expression has been assessed in various human organs, such as respiratory tracts, heart, kidney, ovary, uterus, testis, vagina and placenta, and gastrointestinal system. ${ }^{82}$ Notably, ACE2 is highly expressed in the ovaries. ${ }^{22}$ Published reports suggest that ACE2 is expressed in stromal cells, granulosa cells and oocytes in immature rat ovaries. ${ }^{23}$ ACE2 regulates follicular development and ovulation, regulates luteal angiogenesis and degeneration, and affects the regular changes of endometrial tissue and embryo development. ${ }^{8}$ Significantly, ACE2 plays a regulatory role in reproduction. ${ }^{7}$ Considering these factors, SARS-CoV-2 may interrupt female fertility by attacking ovarian tissue and granulosa cells or damaging endometrial epithelial cells. ${ }^{9}$ Basigin (BSG) is also one of the most crucial receptors for COVID-19 that mediates its entry to host cells. ${ }^{24}$ BSG is expressed not only in the uterus but also in the stroma and granulosa cells of the ovary. ${ }^{24}{ }^{25}$ BSG may play a role during follicle development, corpus luteum formation and embryo implantation. ${ }^{26}$ Besides, COVID-19, impairing the immune system might alter the function of the hypothalamic-pituitary-gonadal axis. ${ }^{2}{ }^{27}$ Sex steroids are potent immune modulators, thus different progesterone and androgen concentrations are likely to influence the immune response and inflammatory outcomes of COVID-19. ${ }^{23}$ Notwithstanding, the magnitude of the association between COVID-19 and female fertility remains unclear. With the overwhelming magnitude of COVID-19 and its worldwide prevalence, the associated health burden, and social and economic costs might be a massive loss around the world.

To our knowledge, there is no systematic review of the potential role of COVID-19 on female fertility. One of the most critical questions that remain to be answered is if or how COVID-19 affects female fertility. Hence, we decided to carry out a systematic review and meta-analysis to improve our understanding of the relationship between COVID-19 and female fertility and facilitate the development of prevention strategies at individual and population levels.

\section{METHODS AND ANALYSIS}

The study will be reported based on the Preferred Reporting Items for Systematic Review and Meta-Analysis Protocols statement ${ }^{28}$ and Meta-analysis of Observational Studies in Epidemiology. ${ }^{29}$ This review's protocol is registered in the International Prospective Register of Systematic Reviews.

\section{Inclusion/exclusion criteria for study selection \\ Study designs and characteristics}

We will include observational studies (cohort studies, cross-sectional studies and case-control studies) and self-controlled case series designs. These studies should also report the impact of COVID-19 on female fertility, including variations in menstrual situation, gonad function, ovarian reserve function, tubal patency and endometrial receptivity. It is not restricted by language, publication status, geography or medical conditions.

\section{Participants}

Female patients in puberty and adulthood (13 years old $\leq$ age $\leq 49$ years old) with COVID-19; women who were not excessively exposed to certain physical, biological, chemical, or environmental factors that affect female fertility, such as lead, cadmium, mercury, pesticides, benzene, toluene and ionising radiation ${ }^{30}$; do not have any gonadal injury caused by high-dose alkylating agent chemotherapy and abdominal/pelvic radiotherapy ${ }^{31}$; and have severe thyroid dysfunction and other diseases that affect female fertility will be excluded. ${ }^{32}$

\section{Exposure/interest}

The exposure factor of interest is infection with SARS-CoV-2, primarily diagnosed by RT-PCR with a nasal swab, tracheal aspirate or bronchoalveolar lavage specimens. ${ }^{33}$

\section{Comparators}

The comparator group will be the population without SARS-CoV-2 infections or with latent SARS-CoV-2 infections.

\section{Outcome measures}

The primary outcome will be at least one of the following indicators of ovarian reserve function: (1) anti-Müllerian hormone $(\mathrm{AMH})$; (2) basal follicle-stimulating hormone (FSH), basal luteinising hormone (LH) or the ratio of FSH/LH; (3) basal oestradiol (E2); (4) serum inhibin-B and (5) antral follicle count (AFC). Secondary outcomes, if any, will be considered: (1) uterine receptivity (endometrial thickness, endometrial morphology, subendometrial blood flow, uterine spiral artery blood flow); (2) oviducts status; (3) menstruation status and (4) other female reproductive dysfunction diseases caused by COVID-19. 


\section{Exclusion criteria}

Studies will be excluded when: (1) the types of research are animal experiments, short surveys and letters; (2) the woman has no potential reproduction before menarche or after menopause; (3) only suspected cases, but not yet confirmed; (4) factors that could affect female fertility, such as severe endocrine diseases or overexposure to certain physical and chemical elements.

\section{Information sources and search strategy}

FL and QZ will search the following databases: Web of Science, PubMed, EMBASE, Cochrane Library, Ovid, EBSCO, WHO COVID-19 Database, Chinese Biomedical Databases, China National Knowledge Internet, Chinese Scientific and Technological Journal Database (VIP) and WanFang Database. The reference lists of selected studies and relevant reviews will be searched to search for relevant articles and identify additional papers not indexed in the databases searched. A systematic search strategy will be employed to identify articles from November 2019 to 30 June 2021, with no language restriction. We will translate non-English articles and conduct update searches before manuscript submission to represent more target populations. The search term will combine Medical Subject Headings with free text to search for concepts such as 'COVID-19' and 'fertility' and 'female'. A detailed search strategy is described in online supplemental table 1 , using PubMed as an example. The search strategies will be adapted to other databases as appropriate and then be checked by another investigator.

\section{Study selection}

Citations identified from the database searches will be imported into EndNote V.X9.1 software, and QY will remove duplicate records. After removing duplicates, QY and LQ will independently screen titles and abstracts for this first level of filtering duplicates. Potentially eligible full-text articles passing the first level of filtering will be independently screened. The studies will be crosschecked according to the predetermined inclusion and exclusion criteria to determine their final inclusion. FL and QZ will examine the included and excluded studies, and verify the reasons for each decision. We will contact the corresponding author for additional information when a study mentions impressive outcomes without providing estimates. If discrepancies arise, we can reach a consensus by discussing or seeking adjudication from a third reviewer.

\section{Data extraction}

Two independent researchers (FL and TW) will use a standardised Excel spreadsheet to independently extract data from the included studies. The outcome measures will be extracted as follows:

\section{Study details}

Title, primary author information, year of publication, journal, study design, country/region, fund source, sample size, age, the period of study and duration of follow-up.

\section{Population characteristics}

Mean baseline age, body weight, height, mean baseline body mass index, race and associated comorbidities.

\section{Exposure}

Diagnostic criteria for COVID-19 as an exposure, number of exposed subjects, duration of disease, details of COVID-19 severity and treatment characteristics.

\section{Comparators}

Definition of unexposed subjects and the number of comparators.

\section{Outcomes}

The primary outcomes are the proportion of women with decreased fertility, the association between COVID-19 and female fertility, or any risk estimate between COVID-19 and female fertility; since ACE2 is most widely expressed in the ovaries, particular attention should be paid to the decreased ovarian reserve function (mean AMH decline, the elevation of basal FSH or LH, a disorder of the FSH/ LH ratio). The secondary outcomes are uterine receptivity (endometrial thickness, endometrial morphology, subendometrial blood flow and uterine spiral artery blood flow), oviducts status and menstrual status.

\section{Quality and bias assessment}

The included studies' methodological quality will be undertaken independently by two reviewers (TW and XL) using appropriate tools. We will use the NewcastleOttawa Scale (NOS) for assessing the quality of cohort and case-control studies. ${ }^{34}$ The NOS includes eight items grouped into three domains: selection of study population, comparability of study groups and exposure (casecontrol studies)/outcome (cohort studies). Each study will be assigned a score of $0-9$. NOS scores greater than 6 are relatively high quality, 5-6 are medium quality and less than 5 are low quality. The Agency for Healthcare Research and Quality (AHRQ) methodology checklist will be applied to evaluate the cross-sectional studies' quality. Using the AHRQ checklist, each study will be judged on 11 items. The quality evaluation is as follows: high quality $=8-$ 11; moderate quality $=4-7$; poor quality $=0-3$. A summary risk of bias table in individual studies will be produced, with a short table to prove each of the judgements in the online supplemental appendix. We will assess the quality of evidence and the strength of recommendations using the Grading of Recommendations, Assessment, Development and Evaluation framework. ${ }^{35}$ Possible discrepancies regarding bias appraisal will be solved by consensus or consulting a third reviewer.

\section{Statistical analysis}

XL and TW will perform statistical analyses using RevMan V.5.3. All statistical tests will be two-tailed, and a p value of $<0.05$ will be considered statistically significant.

\section{Assessment of heterogeneity}

Heterogeneity will be tested for results reported by multiple studies before merging the statistics. We will 
evaluate heterogeneity using the $\mathrm{I}^{2}$ index. If the $\mathrm{I}^{2}$ value is $<50 \%$, a non-substantial level of heterogeneity will be considered, and a fixed-effect model will be applied to the meta-analysis. A random-effects model will be used when the $\mathrm{I}^{2}$ value is $>50 \%$, indicating substantial heterogeneity. We will investigate sources of heterogeneity by using meta-regression analysis and subgroup analysis when substantial heterogeneity is detected.

\section{Data synthesis and analysis}

We will synthesise our results both narratively and quantitatively. Non-quantitative outcomes, such as study characteristics (author, year, study design, country/region and sample size), will be reported descriptively. If considerable heterogeneity cannot be reduced by some methods or the source of heterogeneity cannot be explored using subgroup analysis or regression analysis, we will also conduct a systematic review with descriptive analysis. If there are sufficient studies, we will consider combining outcome data and performing a meta-analysis where appropriate to summarise the evidence for the association between COVID-19 and female fertility.

\section{Publication bias}

Publication bias will be investigated using funnel plots, and Egger's regression test will be applied to statistics when the funnel plots show asymmetry and there are five or more studies available. ${ }^{36}$

\section{Subgroup and sensitivity analyses}

Preplanned subgroup analyses will be conducted to explore statistical heterogeneity. If sufficient data are collected: (1) subgroups based on age (13-35 years and 35-49 years old); (2) the time since fertility decline after COVID-19 infection; to this purpose, three subgroups will be identified: early ( $\leq 30$ days); middle $(<180$ days); late ( $\geq 1$ year); (3) types of fertility decline: ovarian reserve function, uterine receptivity, oviducts status, menstrual status; (4) comorbidities and (5) COVID-19 stage (mild COVID-19 disease and severe COVID-19 disease)-we will remove the included studies from the pooled analyses one by one and perform a sensitivity analysis to assess the robustness of the summary estimate.

\section{Patient and public involvement}

No patient involved.

\section{Ethics and dissemination}

Ethical approval is not required for this study, as it is a systematic review. The results will be disseminated by the publication of the manuscript in a peer-reviewed journal, and national and international presentations.

\section{SUMMARY}

COVID-19 has been a significant public health issue, given its overwhelming magnitude and worldwide prevalence. Direct and indirect evidence suggests that COVID-19 could impair female fertility, which has gained much broader attention.
Female fertility is broadly defined as their reproductive capacity and potential. However, there are no comprehensive reviews to explore the association between COVID-19 and female fertility comprehensively. Hence, we will conduct a systematic review and meta-analysis to improve our understanding of the relationship between COVID-19 and female fertility and facilitate prevention strategies at individual and population levels. The study will also establish the current overall view of COVID-19 and female fertility, given the literature has been updated. By pooling the available evidence on the link of female fertility with COVID-19, promoting fertility preservation in these patients is of high clinical and public health significance.

SARS-CoV-2 may invade target cells by binding to ACE2, thereby affecting female fertility. ACE2, which is widely expressed in ovaries, uterus, vagina and placenta, regulates the levels of angiotensin II (Ang II) and Ang-(1-7) to exert its physiological functions. ${ }^{8}$ ACE2, Ang II and Ang-(1-7) could regulate follicular development and ovulation, regulate corpus luteum angiogenesis and degeneration, and affect endometrial tissue growth. Ovarian reserve is a key determinant of female fertility. Diminished ovarian reserve could affect fecundity by reducing egg quality. ${ }^{37}$ Besides, ACE2 is highly expressed in the ovaries. Therefore, the ovarian reserve function should be the primary observation indicator for the impact of COVID-19 on female fertility. Routinely performed markers of the ovarian reserve include a basal FSH or LH concentration, E2, AMH and assessment of AFC..$^{39}$ We will use endometrial receptivity, fallopian tube status and menstrual conditions as secondary indicators. Furthermore, subgroup analyses and sensitivity analyses will be conducted to explore heterogeneity, such as age, follow-up time, the type of fertility decline, comorbidities and severity of illness. It is generally accepted that fertility starts at (approximately) 13 years of age, and women begin to become infertile at age 49 years. ${ }^{40}$ With ageing, the female fertility naturally declines. The decline in fertility in women aged 30-35 years is slow and steady; however, the decline accelerates after the age of 35 years due to decreases in the ovarian reserve and oocyte quality. ${ }^{41}$ The subgroup analysis will assess the impact of COVID-19 on the fertility of women aged $<35$ years and $>35$ years.

The majority of the relevant studies may come from cohort studies, cross-sectional studies, case-control studies and self-controlled case series designs. Nevertheless, we will conduct a systematic review and meta-analysis based on the existing evidence to explore the immediate, medium-term and long-term effects of COVID-19 on female fertility. It is of great significance to plan and take action to protect female fertility when there is a negative impact on fertility.

Contributors The study concept was developed by FL. The manuscript of the protocol was drafted by FL and critically revised by $Q Z$ and XL. HL developed and provided feedback for all sections of the review protocol and approved the final manuscript. The search strategy was developed by FL and QZ. Study selection will be performed by QY and LQ. Data extraction and quality assessment will be performed by FL and TW, with $\mathrm{QL}$ as a third party in case of disagreements. All authors have approved the final version of the manuscript. 
Funding This study is financially supported by the Ministry of Science and Technology of the People's Republic of China (grant number: 2018YFC1704305). Competing interests None declared.

Patient consent for publication Not required.

Provenance and peer review Not commissioned; externally peer reviewed.

Supplemental material This content has been supplied by the author(s). It has not been vetted by BMJ Publishing Group Limited (BMJ) and may not have been peer-reviewed. Any opinions or recommendations discussed are solely those of the author(s) and are not endorsed by BMJ. BMJ disclaims all liability and responsibility arising from any reliance placed on the content. Where the content includes any translated material, BMJ does not warrant the accuracy and reliability of the translations (including but not limited to local regulations, clinical guidelines, terminology, drug names and drug dosages), and is not responsible for any error and/or omissions arising from translation and adaptation or otherwise.

Open access This is an open access article distributed in accordance with the Creative Commons Attribution Non Commercial (CC BY-NC 4.0) license, which permits others to distribute, remix, adapt, build upon this work non-commercially, and license their derivative works on different terms, provided the original work is properly cited, appropriate credit is given, any changes made indicated, and the use is non-commercial. See: http://creativecommons.org/licenses/by-nc/4.0/.

\section{ORCID iD}

Hua Lu http://orcid.org/0000-0002-2709-222X

\section{REFERENCES}

1 World Health Organization. Coronavirus disease 2019 (COVID-2019) situation Report-49, 2020. Available: https://www.who.int/ emergencies/diseases/novel-coronavirus-2019 [Accessed 2 Sep 2020].

2 Yang L, Liu S, Liu J, et al. COVID-19: immunopathogenesis and immunotherapeutics. Signal Transduct Target Ther 2020;5:128.

3 Cao X. COVID-19: immunopathology and its implications for therapy. Nat Rev Immunol 2020;20:269-70.

$4 \mathrm{XW} X, \mathrm{XX}$ W, Jiang XG. Clinical findings in a group of patients infected with the 2019 novel coronavirus (SARS-Cov-2) outside of Wuhan, China: retrospective case series. BMJ 2020;368.

5 Gupta A, Madhavan MV, Sehgal K, et al. Extrapulmonary manifestations of COVID-19. Nat Med 2020;26:1017-32.

6 Aitken RJ. COVID-19 and human spermatozoa-Potential risks for infertility and sexual transmission? Andrology 2021;9:48-52.

$7 \mathrm{Fu}$ J, Zhou B, Zhang L, et al. Expressions and significances of the angiotensin-converting enzyme 2 gene, the receptor of SARS-CoV-2 for COVID-19. Mol Biol Rep 2020;47:4383-92.

8 Jing Y, Run-Qian L, Hao-Ran W, et al. Potential influence of COVID-19/ACE2 on the female reproductive system. Mol Hum Reprod 2020;26:367-73.

9 Li R, Yin T, Fang F, et al. Potential risks of SARS-CoV-2 infection on reproductive health. Reprod Biomed Online 2020;41:89-95.

10 Aassve A, Cavalli N, Mencarini L, et al. The COVID-19 pandemic and human fertility. Science 2020;369:370-1.

11 Li G, Tang D, Song B, et al. Impact of the COVID-19 pandemic on partner relationships and sexual and reproductive health: crosssectional, online survey study. J Med Internet Res 2020;22:e20961.

12 Sills ES, Wood SH. An experimental model for Peri-conceptual COVID-19 pregnancy loss and proposed interventions to optimize outcomes. Int J Mol Cell Med 2020;9:180-7.

13 Schwartz DA, Graham AL. Potential maternal and infant outcomes from (Wuhan) coronavirus 2019-nCoV infecting pregnant women: lessons from SARS, MERS, and other human coronavirus infections. Viruses 2020;12:194.

14 Hsu AL, Guan M, Johannesen E, et al. Placental SARS-CoV-2 in a pregnant woman with mild COVID-19 disease. J Med Virol 2021;93:1038-1044.

15 Li W, Moore MJ, Vasilieva N, et al. Angiotensin-Converting enzyme 2 is a functional receptor for the SARS coronavirus. Nature 2003;426:450-4

16 Lukassen S, Chua RL, Trefzer T, et al. SARS-CoV-2 receptor ACE2 and TMPRSS2 are primarily expressed in bronchial transient secretory cells. Embo J 2020;39:e105114.
17 Hikmet F, Méar L, Edvinsson Åsa, et al. The protein expression profile of ACE2 in human tissues. Mol Syst Biol 2020;16:e9610.

18 Zhou Y, Vedantham P, Lu K, et al. Protease inhibitors targeting coronavirus and filovirus entry. Antiviral Res 2015;116:76-84.

19 Matsuyama S, Nagata N, Shirato K, et al. Efficient activation of the severe acute respiratory syndrome coronavirus spike protein by the transmembrane protease TMPRSS2. J Virol 2010;84:12658-64.

20 Hoffmann M, Kleine-Weber H, Schroeder S, et al. SARS-CoV-2 cell entry depends on ACE2 and TMPRSS2 and is blocked by a clinically proven protease inhibitor. Cell 2020;181:271-80.

21 Lippi G, Lavie CJ, Henry BM, et al. Do genetic polymorphisms in angiotensin converting enzyme 2 (ACE2) gene play a role in coronavirus disease 2019 (COVID-19)? Clin Chem Lab Med 2020;58:1415-22.

22 Ace2 gene (protein coding). Available: https://www.genecards. org/cgi-bin/carddisp.pl?gene=ACE2\&keywords=ACE2\#protein expression

23 Pereira VM, Reis FM, Santos RAS, et al. Gonadotropin stimulation increases the expression of angiotensin-(1--7) and MAS receptor in the rat ovary. Reprod Sci 2009;16:1165-74.

24 Mahdian S, Shahhoseini M, Moini A. COVID-19 mediated by basigin can affect male and female fertility. Int J Fertil Steril 2020;14:262-3.

25 Chen L, Bi J, Nakai M, et al. Expression of basigin in reproductive tissues of estrogen receptor-\{alpha\} or -\{beta\} null mice. Reproduction 2010;139:1057-66.

26 Chang $\mathrm{H}, \mathrm{Ni} \mathrm{H}, \mathrm{Ma} \mathrm{X}-\mathrm{H}$, et al. Basigin expression and regulation in mouse ovary during the sexual maturation and development of corpus luteum. Mol Reprod Dev 2004;68:135-41.

27 Mauvais-Jarvis F, Klein SL, Levin ER. Estradiol, progesterone, immunomodulation, and COVID-19 outcomes. Endocrinology 2020;161:bqaa127.

28 Shamseer L, Moher D, Clarke M, et al. Preferred reporting items for systematic review and meta-analysis protocols (PRISMA-P) 2015: elaboration and explanation. BMJ 2015;350:g7647.

29 Stroup DF, Berlin JA, Morton SC, et al. Meta-Analysis of observational studies in epidemiology: a proposal for reporting. meta-analysis of observational studies in epidemiology (moose) group. JAMA 2000;283:2008-12.

30 Kumar S, Sharma A, Kshetrimayum C. Environmental \& occupational exposure \& female reproductive dysfunction. Indian J Med Res 2019;150:532-45.

31 van Dorp W, Haupt R, Anderson RA, et al. Reproductive function and outcomes in female survivors of childhood, adolescent, and young adult cancer: a review. J Clin Oncol 2018;36:2169-80.

32 Korevaar TIM, Mínguez-Alarcón L, Messerlian C, et al. Association of thyroid function and autoimmunity with ovarian reserve in women seeking infertility care. Thyroid 2018;28:1349-58.

33 Pascarella G, Strumia A, Piliego C, et al. COVID-19 diagnosis and management: a comprehensive review. J Intern Med 2020;288:192-206.

34 Wells GA, Shea B, O'Connell D. The Newcastle-Ottawa scale (NOS) for assessing the quality of Nonrandomised studies in Metaanalyses. Ottawa: Ottawa Hospital Research Institute, 2019. http://www.ohri. $\mathrm{ca} /$ programs/clinical_epidemiology/oxford.asp

35 Guyatt GH, Oxman AD, Vist GE, et al. Grade: an emerging consensus on rating quality of evidence and strength of recommendations. $B M J$ 2008;336:924-6.

36 Egger M, Davey Smith G, Schneider M, et al. Bias in meta-analysis detected by a simple, graphical test. BMJ 1997;315:629-34.

37 Steiner AZ, Pritchard D, Stanczyk FZ, et al. Association between biomarkers of ovarian reserve and infertility among older women of reproductive age. JAMA 2017;318:1367-76.

38 Tal R, Seifer DB. Ovarian reserve testing: a user's guide. Am J Obstet Gynecol 2017;217:129-40.

39 Johnson NP, Bagrie EM, Coomarasamy A, et al. Ovarian reserve tests for predicting fertility outcomes for assisted reproductive technology: the International systematic collaboration of ovarian reserve evaluation protocol for a systematic review of ovarian reserve test accuracy. BJOG 2006;113:1472-80.

40 Jensen RE, Martins N, Parks MM. Public perception of female fertility: initial fertility, peak fertility, and age-related infertility among U.S. adults. Arch Sex Behav 2018;47:1507-16.

41 Ahmed TA, Ahmed SM, El-Gammal Z, et al. Oocyte aging: the role of cellular and environmental factors and impact on female fertility. Adv Exp Med Biol 2020;1247:109-23. 